\title{
Planejamento da aposentadoria: Adaptação brasileira da PRePS e influência de estilos de tomada de decisão
}

Retirement Planning: Brazilian adaptation of PRePS and the influence of decision-making styles Planificación de la jubilación: La adaptación brasileña de la PRePS e influencia de los estilos

\author{
Julia Carolina RAFALSKI ${ }^{1}$ \\ Alexsandro Luiz DE ANDRADE \\ Universidade Federal do Espírito Santo, Vitória, ES, Brasil
}

Resumo A temática do planejamento da aposentadoria tem crescido em importância com o aumento da expectativa de vida e a previsão de déficit previdenciário no Brasil. A fim de fornecer instrumentos que possibilitem a avaliação de aspectos preparatórios para a aposentadoria, este artigo teve como objetivos: (a) adaptar a Process of Retirement Planning Scale (PRePS) para o português brasileiro e (b) verificar a influência de estilos de tomada de decisão no planejamento da aposentadoria. Participaram do estudo 1.290 trabalhadores com idades entre 18 e 69 anos $(M=37,06 ; D P=10,5)$, sendo a maioria homens ( $N=944 ; 73,3 \%)$. A versão brasileira da PRePS foi chamada de Escala de Processo de Planejamento da Aposentadoria (EPP1A), e os resultados mostraram que o estilo de tomada de decisão racional influencia positiva e significativamente os quatro âmbitos do planejamento da aposentadoria: saúde, finanças, estilo de vida e psicossocial. Os estilos dependente, intuitivo, espontâneo e evitativo também se mostraram preditores significativos em diferentes dimensões.

Palavras-chave:

Planejamento da aposentadoria; tomada de decisão; escalas psicológicas.

Abstract Retirement planning has grown in importance with the rising life expectancy and the forecasted social security deficit in Brazil. In order to provide instruments to evaluate aspects of preparation for retirement, this article aimed to: (a) adapt the Process of Retirement Planning Scale (PRePS) to Brazilian Portuguese, and (b) verify the influence of decision-making styles on retirement planning. Participants were 1,290 workers between 18 and 69 years of age $(M=37.06 ; S D=10.5)$ mostly men $(N=944 ; 73.3 \%)$. The Brazilian version of PRePS received the name Escala de Processo de Planejamento da Aposentadoria (EPP1A), and the results of this study showed that rational decision-making style positively and significantly influences all 4 retirement planning spheres: health, finances, life style, and psychosocial. dependent, intuitive, spontaneous, and avoidant styles also proved to be significant predictors in different dimensions.

Keywords:

Retirement planning; decision-making styles; psychological scales.

Resumen El tema de la planificación de la jubilación ha crecido en con el aumento de la expectativa de vida y el prognóstico de déficit en las pensiones de jubilación en Brasil. Con el fin de proporcionar herramientas que permitan la evaluación de los aspectos de preparación para la jubilación, este artículo pretende: (a) adaptar la Process of Retirement Planning Scale (PRePS) para portugués brasileño y, (b) investigar la influencia de los estilos de toma de decisiones en la planificación de la jubilación. Participaron del estudio 1290 trabajadores con edades entre 18 y 69 años $(M=37,06 ; S D=10,5)$, la mayoría hombres $(N=944 ; 73,3 \%)$. La versión brasileña de PREPS fue llamada Escala de Processo de Planejamento da Aposentadoria (EPP1A), y los resultados mostraron que el estilo de toma de decisiones racional influencia positiva y significativamente los 4 áreas de planificación de la 
jubilación: salud, finanzas, estilo de vida y psicosocial. Estilos dependientes, intuitivos, espontáneos y de evitación también fueron predictores significativos en diferentes dimensiones.

Palabras-clave:

Planificación de la jubilación; toma de decisiones; escalas psicológicas.

A

temática da aposentadoria vem ganhando espaço nas pesquisas brasileiras e na mídia principalmente devido à situação previdenciária conturbada e à crescente expectativa de vida dos habitantes do país (Antunes, Soares, \& Silva, 2013). O número de trabalhos sobre planejamento (p. ex., Menezes \& França, 2012) tem crescido, assim como a literatura sobre os programas de preparação/orientação para a aposentadoria (PPA/POA) (Costa \& Soares, 2009; Muniz, 1996), acompanhando a legislação que estimula as empresas a fornecerem esse tipo de suporte aos seus funcionários (Lei 8.842/94).

O planejamento da aposentadoria atua de forma a prevenir problemas de saúde (Noone, Stephens, \& Alpass, 2009), ampliar a capacidade de economizar e administrar o tempo (Wang \& Shultz, 2010), além de desenvolver expectativas para o futuro (Adams \& Rau, 2011). O contexto socioeconômico atual endossa a autonomia e a preparação cada vez mais como ação advinda do trabalhador (Rowlingson, 2002), mesmo que o sistema previdenciário brasileiro tenha como base a comunidade e a repartição simples dos dividendos (Marques, Batich, \& Mendes, 2003). Preparar-se para a aposentadoria envolve o planejamento e a tomada de decisão em favor de comportamentos facilitadores (Leandro-França, Murta, \& Villa, 2014) que promovam confiança para se aposentar (Kim, Kwon, \& Anderson, 2005).

Noone, Stephens e Alpass (2010) desenvolveram a Process of Retirement Planning Scale (PRePS) para avaliar o planejamento da aposentadoria considerando a teoria de Friedman e Scholnick (1997), que expõe que planejar envolve: (a) o conhecimento sobre o assunto (representações), (b) a construção de objetivos, (c) a tomada de decisão e (d) os comportamentos de planejamento - quatro domínios do planejamento da aposentadoria. Os autores avaliaram o processo de planejamento em quatro escalas - saúde, finanças, estilo de vida e psicossocial - que abarcam os quatro domínios (representações, objetivos, decisão e comportamentos). Juntos, esses domínios abrangem o processo de planejamento da aposentadoria de forma compreensiva, entendendo as diferentes esferas que impactam a vida dos indivíduos (Topa, Moriano, Depolo, Alcover, \& Morales, 2009).

O processo da aposentadoria trata a fase de planejamento como imediatamente anterior à transição da saída do trabalho, considerando que o processo em si se inicia muito antes dessa passagem (Adams \& Rau, 2011; Wang, 2012). Para Wang e Shultz (2010), a decisão de se aposentar é antecedente ao planejamento, na medida em que fatores pessoais (p. ex., idade), circunstâncias (p. ex., vínculo empregatício) e recursos disponíveis (p. ex., plano de previdência, habilidades sociais) podem alterar a preparação para a aposentadoria. A decisão possui papel relevante: a aposentadoria involuntária/forçada tem impacto significativo e negativo no bem-estar dos idosos (Bonsang \& Klein, 2012), enquanto ter esse controle amplia a autonomia na velhice (Moen, 1996).

Ainda que não esteja claro se percepções negativas ou traços de personalidade negativos (como neuroticismo) influenciam a decisão de se aposentar (Feldman \& Beehr, 2011), existem pesquisas que procuram compreender o quanto das decisões é influenciado por fatores individuais e o quanto é relegado ao contexto (Appelt, Milch, Handgraaf, \& Weber, 2011). Os estilos de tomada de decisão são incluídos na porção individual da avaliação e escolha de eventos, sendo definidos como hábitos de reação, e não traços de personalidade (Scott \& Bruce, 1995). São divididos em cinco dimensões não excludentes: (a) estilo racional: descreve pessoas que utilizam o pensamento lógico e sistemático para tomar decisões, (b) estilo espontâneo: caracteriza pessoas que preferem tomar decisões de acordo com o desenvolvimento dos problemas e do contexto, (c) estilo dependente: abarca pessoas que preferem contar com pares (amigos, familiares, etc.) ou pessoas especializadas para aconselhá-los, (d) estilo evitativo: descreve pessoas que se esquivam de tomar decisões e adiam essa ação tanto quanto possível e (e) estilo intuitivo: caracteriza pessoas que usam seus sentimentos e emoções para tomar decisões (Scott \& Bruce, 1995).

Pesquisas utilizam os estilos de decisão para entender diferentes fenômenos, como estresse (Allwood \& Salo, 2012), satisfação com a vida e autoestima baseada na performance (Salo \& Allwood, 2011) e liderança (Hariri, Monypenny, \& Prideaux, 2013). Os resultados dos trabalhos citados apontam a relação entre o estilo racional e variáveis positivas (como menos estresse, maior satisfação com a vida e maiores níveis de liderança), enquanto os estilos dependente e evitativo se relacionam com variáveis mais negativas (como maior percepção de estresse e menor qualidade de sono, menor satisfação com a vida e menor liderança). 
O objetivo deste trabalho é, primeiramente, adaptar a PRePS para o português brasileiro. Em seguida, serão realizados procedimentos estatísticos para verificar o impacto dos estilos de tomada de decisão no planejamento da aposentadoria.

\section{MÉTODO}

\section{Tradução e adaptação da PRePS}

A PRePS foi desenvolvida por Noone et al. (2010) para avaliar quatro domínios do planejamento da aposentadoria: saúde, finanças, psicossocial e estilo de vida. A escala original conta com 52 itens agrupados nos quatro domínios considerados mais relevantes para o planejamento em relação à vida do indivíduo e foi validada em população de neozelandeses ( $N=1449 ; M=53,4$ anos; $D P=2,89 ; 55 \%$ mulheres). Possui escala de resposta do tipo Likert com 5 pontos, variando entre "definitivamente falso para mim" e "definitivamente verdadeiro para mim”. Os itens são organizados em cada um dos domínios (escalas) em relação às quatro fases do planejamento: representações/percepções, objetivos, decisão e planejamento. As subescalas de objetivos são formadas por item único. Dessa forma, a escala de planejamento de saúde, por exemplo, conta com apenas um item de objetivo de saúde, itens de representações de saúde, itens de decisão para saúde e itens de planejamento de saúde em número variável. Os demais domínios possuem o mesmo desenho.

\section{Procedimentos de tradução e adaptação - Evidências de validade de construto}

Conforme resolução 466/2012 do Conselho Nacional de Saúde, o estudo foi analisado e aprovado pelo parecer número 473.624 via avaliação do Comitê de Ética em Pesquisa com Seres Humanos (CEPSH) da Universidade Federal do Espírito Santo. Em 2013, obteve-se permissão dos autores para realizar a adaptação da escala original, feita pela primeira autora e por dois doutores em psicologia bilíngues e especialistas na área de carreira. Os procedimentos de tradução e retradução foram realizados conforme indicação de Hambleton e Zenisky (2011) e buscaram fornecer adaptações semânticas e culturais para que a mensagem a ser transmitida pelo item fosse fidedigna à população-alvo (Van Ommeren et al., 1999).

A primeira tradução foi realizada pela primeira autora e em sequência foi feita a tradução reversa pelos dois juízes, do português para o inglês e vice-versa. As traduções foram confrontadas para verificar a adequação cultural e semântica. Todos os 52 itens da PRePS foram traduzidos. Dois itens adicionais foram apresentados a partir dos originais por sugestão dos juízes, somando, então, 54 itens. A escala traduzida com 54 itens foi considerada a versão preliminar.

Após a tradução, a escala foi aplicada em seis trabalhadores (três deles mulheres) com idades acima de 35 anos para avaliar a adequação semântica utilizando um questionário que continha outras escalas e questões demográficas. Os participantes avaliaram os itens quanto a sua facilidade de compreensão e adequação de termos à cultura brasileira. Não foram sugeridas modificações quanto à forma de escrita dos itens e a escala permaneceu em sua versão preliminar.

\section{Levantamento dos indicadores empíricos de validade e precisão}

\section{Participantes}

Participaram desta etapa 982 trabalhadores, divididos randomicamente em dois grupos: um destinado à análise fatorial confirmatória (grupo 1) e um destinado à análise fatorial exploratória (grupo 2). Ambos os grupos possuíam 491 participantes, com 65,3\% homens. As idades variaram entre 18 e 70 anos, sendo a média do grupo 1 de 39,5 anos $(D P=10,5)$ e do grupo 2, 40,3 anos $(D P=10,5)$. Em ambos os grupos predominaram participantes atuantes em empresas públicas ( $N=376 ; 71,6 \%$ grupo 1 e $N=350 ; 69,9 \%$ grupo 2) e com, pelo menos, ensino superior completo ( $N=370 ; 75,6 \%$ grupo 1 e $N=362 ; 74 \%$ grupo 2$)$. O grupo total de 982 trabalhadores possui média de idade de 40,1 anos ( $D P=10,5)$, com $N=640 ; 65,1 \%$ do sexo masculino. Também predominaram participantes com, pelo menos, superior completo $(N=732 ; 74,8 \%)$ e advindos de empresas públicas $(N=726 ; 70,8 \%)$. A maioria dos participantes $(N=919 ; 93,6 \%)$ foi proveniente de um estado da região Sudeste.

A amostra da etapa que avalia a influência dos estilos de decisão no planejamento da aposentadoria compreende participantes presentes na etapa de validação da escala e novos participantes advindos da mesma 
coleta de dados. Participaram 1.290 trabalhadores de empresas públicas ( $N=1176 ; 91,2 \%)$ e privadas ( $N=114$; $5,7 \%)$. As idades variaram de 18 a 69 anos $(M=37,06 ; D P=10,5)$, com maioria homens $(N=944 ; 73,3 \%)$, pessoas casadas $(N=800 ; 62,1 \%)$ e com, pelo menos, ensino superior completo $(N=920 ; 71,5 \%)$. A maioria $(N=1249$; $96,8 \%)$ era proveniente de um estado da região Sudeste.

\section{Instrumentos}

Para a coleta de dados, foi utilizada a versão preliminar da PRePS com 54 itens. Foi elaborado um questionário sociodemográfico com questões para descrever os participantes: idade, sexo, estado civil, "empresa em que trabalha possui orientação para aposentadoria?", "pretende retornar ao trabalho após se aposentar?”, entre outras.

Para compreender os estilos de decisão, foi utilizado o Inventário Geral de Estilos de Tomada de Decisão (IGETD), adaptado para o português por Rafalski e De Andrade (2014) a partir do original em inglês (Scott \& Bruce, 1995). O IGETD é um inventário com escala de resposta tipo Likert de 5 pontos (1-discordo fortemente a 5-concordo fortemente) e conta com 25 itens divididos em 5 dimensões: racional, evitativo, espontâneo, intuitivo e dependente.

\section{Procedimentos de coleta e análise de dados}

A coleta de dados foi realizada após concessão de permissão das empresas públicas e privadas. Os trabalhadores foram convidados a participar voluntariamente, informados quanto ao sigilo e assinaram o Termo de Consentimento Livre e Esclarecido em duas vias. A coleta foi realizada presencialmente e on-line, de acordo com a preferência de cada empresa.

Os dados foram analisados com auxílio dos pacotes estatísticos SPSS (Statistical Package for the Social Sciences) e AMOS (Analysis of Moment Structures). Utilizaram-se os indicadores de ajuste para os modelos confirmatórios: qui-quadrado ponderado (razão do qui-quadrado por graus de liberdade, $\chi^{2} / g l$ ) menor que 3 ; goodness of fit index $(G F I)$ e comparative fit index $(C F I)$, valores próximos a 1; e root mean square error of approximation (RMSEA), valor inferior a 0,08 (Byrne, 2010). Com o SPSS, recorreu-se à análise de componentes principais (principal components analysis - $P C A$ ) e à análise dos principais eixos fatoriais (principal axis factoring - $P A F$ ) utilizando a rotação direct oblimin, além dos índices de confiabilidade alfa de Cronbach.

Para as análises concernentes aos estilos de tomada de decisão, foram feitas regressões lineares hierárquicas utilizando o software SPSS. Foi utilizado o método Enter, que elimina o viés de overfitting - que define a exigência em demasia dos dados para produzir predições a partir das correlações entre as variáveis (Babyak, 2004) -, além de verificar a influência preditiva das variáveis sem fixá-las em modelos estáticos, mas em modelos teóricos. Regressões hierárquicas são úteis para eliminar possíveis vieses de coleta e de falta de randomização de participantes, além de controlarem efeitos primários, como variáveis de características dos respondentes (Hox, 1994). Desse modo, é possível concluir com maior segurança o papel das variáveis significativas sobre o fenômeno a ser explicado.

\section{Indicadores empíricos de validade e precisão}

A fim de confirmar a estrutura da PRePS no Brasil, foram testados, com o software AMOS, primeiramente os modelos originais para cada uma das quatro escalas: finanças, psicossocial, saúde e estilo de vida. Conforme procedimentos do artigo original, a subescala de objetivos para a aposentadoria não integra o modelo confirmatório, pois é formada por um item em cada domínio. Foram testados quatro modelos, nomeados "modelos originais", correspondentes ao artigo original, contando com itens relativos às representações, às decisões e à preparação para a aposentadoria.

Os índices de adequação dos modelos originais de todas as escalas sugerem que as estruturas fatoriais originais não são adequadas para os dados brasileiros, cujos resultados são apresentados na Tabela 1. Foram encontradas elevadas associações entre itens de diferentes subescalas em todas as dimensões, como, por exemplo, "Penso muito sobre minha situação econômica na aposentadoria", um item de representação, e "Se eu fosse forçado a me aposentar hoje teria economias suficientes para lidar bem com a aposentadoria”, que corresponde aos itens de preparação.

Em consideração aos resultados dos modelos originais, foram criados novos modelos, após a verificação de favoráveis índices para fatorabilidade das matrizes (todos significativos a $p<0,001$ ). Os "modelos novos" 
e os "modelos adaptados" foram obtidos a partir da análise fatorial exploratória por meio dos componentes principais e, em seguida, pelo método dos eixos principais. Semelhante ao realizado nos modelos originais, foram elaborados quatro modelos novos e quatro modelos adaptados, um para cada dimensão/escala (saúde, finanças, estilo de vida e psicossocial).

Para os modelos novos, foram fatorados todos os itens da escala original, incluindo o item de objetivos, em cada uma das quatro escalas. Utilizou-se como base o entendimento de Pasquali (2009), que expõe que todas as variáveis comportamentais devem constar em análise fatorial para fazerem parte de um conjunto observável. Isso resultou em 4 subfatores para finanças, 2 subfatores para estilo de vida, 4 subfatores para psicossocial e 3 subfatores para saúde.

Para os modelos adaptados, foram fatorados todos os itens exceto o de objetivos, seguindo a indicação de Noone et al.(2010). Como resultados, finanças obteve 4 subfatores; estilo de vida, 4; psicossocial, 3; e saúde, 3. Ainda que alguns dos modelos novos e adaptados se assemelhem, as distribuições dos itens foram diferenciadas, uma vez que o item objetivos produziu modificações nas cargas fatoriais e nos carregamentos dos itens em cada fator extraído. Os índices de adequação dos modelos novos e adaptados estão disponíveis na Tabela 1, bem como índices comparativos entre os modelos.

TABELA 1. Testes de estruturas fatoriais das dimensões da Escala de Processo de Planejamento da Aposentadoria (EPPIA)

\begin{tabular}{|c|c|c|c|c|c|c|c|c|c|c|}
\hline & Modelos & $g l$ & $\chi^{2}$ & $x^{2 / g I}$ & CFI & GFI & RMSEA & $\Delta \chi^{2}$ & $\Delta g l$ & $\Delta \boldsymbol{C F I}$ \\
\hline \multirow{3}{*}{ Finanças } & $\mathrm{MO}$ & 76 & $373,794^{\star *}$ & 4,918 & 0,886 & 0,907 & 0,089 & - & - & - \\
\hline & MN & 54 & $250,248^{* *}$ & 4,634 & 0,916 & 0,928 & 0,086 & 123,546 & 22 & $-0,03$ \\
\hline & MA & 37 & $131,621^{\star *}$ & 3,557 & 0,949 & 0,953 & 0,072 & 242,173 & 39 & $-0,063$ \\
\hline \multirow{3}{*}{ Estilo de vida } & $\mathrm{MO}$ & 27 & $99,072^{\star \star}$ & 3,670 & 0,916 & 0,960 & 0,074 & - & - & - \\
\hline & MN & 30 & $163,619^{\star \star}$ & 5,454 & 0,870 & 0,940 & 0,095 & $-64,547$ & -3 & 0,046 \\
\hline & MA & 14 & $44,652^{\star \star}$ & 3,186 & 0,952 & 0,978 & 0,067 & 54,42 & 13 & $-0,036$ \\
\hline \multirow{3}{*}{ Psicossocial } & MO & 59 & 324,537 & 5,501 & 0,885 & 0,894 & 0,096 & - & - & - \\
\hline & MN & 44 & $197,617^{\star \star}$ & 4,491 & 0,915 & 0,936 & 0,084 & 126,92 & 15 & $-0,03$ \\
\hline & MA & 39 & $118,412^{\star \star}$ & 3,036 & 0,945 & 0,957 & 0,064 & 206,125 & 20 & $-0,06$ \\
\hline \multirow{3}{*}{ Saúde } & $\mathrm{MO}$ & 30 & $177,120^{\star *}$ & 5,904 & 0,868 & 0,927 & 0,100 & - & - & - \\
\hline & MN & 24 & $139,662^{\star \star}$ & 5,819 & 0,889 & 0,939 & 0,099 & 37,458 & 6 & $-0,021$ \\
\hline & MA & 23 & $49,266^{\star \star}$ & 2,142 & 0,969 & 0,979 & 0,048 & 127,854 & 7 & $-0,101$ \\
\hline
\end{tabular}

Nota. ${ }^{* *} p<0,001, N=982$. MO: modelo original; MN: modelo novo; MA: modelo adaptado.

Os resultados apontam que adicionar a variável de objetivos ao desenho estrutural dos modelos novos não produziu melhorias nos índices de ajuste em comparação aos modelos originais. O modelo adaptado obteve o melhor ajuste em todas as dimensões, tendo sido reespecificado a partir do modelo original, e escolhido como versão em português/Brasil da escala.

A PRePS, em sua versão brasileira, foi nomeada Escala de Processo de Planejamento da Aposentadoria (EPP1A) e conta com 44 itens, divididos em 4 escalas: planejamento de saúde $(\alpha=0,65$; Tabela 2$)$, planejamento financeiro ( $\alpha=0,79$; Tabela 3$)$, planejamento de estilo de vida ( $\alpha=0,62$; Tabela 4$)$ e planejamento psicossocial ( $\alpha=0,83$; Tabela 5). Cargas fatoriais e alfas para cada subescala estão disponíveis em cada tabela, desenhada de acordo com o artigo original. 
TABELA 2. Estrutura fatorial da EPPIA - Subescala planejamento de saúde

Itens

$\alpha$

5. Converso frequentemente com minha família sobre nossa condição de saúde futura.

14. Frequentemente comparo minha saúde atual com a forma que gostaria que ela fosse no futuro.

2. Costumo fazer exames médicos para câncer, diabetes e doenças cardíacas.

37. Considero importante começar a cuidar da minha saúde agora em vez de lidar com

¿ problemas mais perto da aposentadoria.

ㄴ. 22. Tenho um entendimento claro da importância da saúde para pessoas mais velhas.

26. Considero que é muito cedo para pensar na minha condição de saúde durante a aposentadoria.

3. Procuro evitar comportamentos não saudáveis.

47. Procuro me alimentar apenas com alimentos que beneficiarão minha saúde em longo prazo.

7. Procuro me manter fisicamente ativo (caminhando regularmente, praticando atividades físicas, jogando futebol...).
0,79

0,54

0,47

51. Possuo metas específicas para minha saúde em longo prazo

$3,58^{*}$

Nota. N=982, Principal Axis Fatorial, método direct oblimin. 1-Representações; 2-Decisões de tempo; 3-Decisões de pares; e 4-Preparação. *Itens da escala de objetivos, com média do item.

TABELA 3. Estrutura fatorial da EPPIA - Subescala planejamento financeiro

\begin{tabular}{|c|c|c|c|c|c|c|}
\hline & Itens & $\alpha$ & 1 & 2 & 3 & 4 \\
\hline \multirow{12}{*}{ 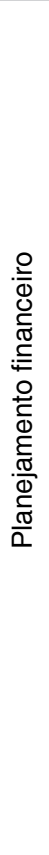 } & $\begin{array}{l}\text { 4. Comparo frequentemente minha posição financeira atual com a posição financeira que } \\
\text { eu gostaria de ter na aposentadoria. }\end{array}$ & & 0,66 & & & \\
\hline & 46. Penso muito sobre minha futura situação econômica. & 0,60 & 0,65 & & & \\
\hline & 32. Vale a pena economizar dinheiro para a aposentadoria. & & & 0,70 & & \\
\hline & $\begin{array}{l}\text { 13. Considero importante começar a economizar hoje em vez de lidar com questões } \\
\text { financeiras mais perto da aposentadoria. }\end{array}$ & 0,66 & & 0,67 & & \\
\hline & $\begin{array}{l}\text { 45. Falo frequentemente com a minha família sobre questões financeiras para aposen- } \\
\text { tados. }\end{array}$ & & & & 0,66 & \\
\hline & $\begin{array}{l}\text { 41. Sei que pessoas da minha faixa etária estão se preparando financeiramente para a } \\
\text { aposentadoria. }\end{array}$ & 0,52 & & & 0,49 & \\
\hline & $\begin{array}{l}\text { 28. Quando eu me aposentar, terei renda suficiente para assegurar o padrão de vida que } \\
\text { quero na aposentadoria. }\end{array}$ & & & & & 0,95 \\
\hline & $\begin{array}{l}\text { 17. Quando eu me aposentar, terei renda suficiente para assegurar o padrão de vida } \\
\text { que tenho hoje. }\end{array}$ & & & & & 0,90 \\
\hline & 34. Quando eu me aposentar, terei dinheiro suficiente para pagar despesas inesperadas. & & & & & 0,85 \\
\hline & $\begin{array}{l}\text { 10. Se eu fosse forçado a me aposentar com } 65 \text { anos, teria economias suficientes para } \\
\text { lidar bem com a aposentadoria. }\end{array}$ & & & & & 0,66 \\
\hline & 15. Quando eu me aposentar, terei uma casa sem financiamento. & 0,85 & & & & 0,36 \\
\hline & 25. Tenho metas claras sobre a condição financeira que quero ter na aposentadoria. & & & $3,44^{*}$ & & \\
\hline
\end{tabular}

Nota. N=982, Principal Axis Fatorial, método direct oblimin. 1-Representações; 2-Decisões de tempo; 3-Decisões de pares; e 4-Preparação. *Itens da escala de objetivos, com média do item. 


\section{Itens}

$\alpha$

1. Tenho pensado muito sobre como passarei meu tempo na aposentadoria.

0,70

16. Frequentemente comparo como passo meu tempo agora com a forma que gostaria de passá-lo na aposentadoria.

$0,63 \quad 0,64$

35. Eu prefiro decidir o que fazer com meu tempo quando me aposentar em vez de pen-

은 sar sobre isso agora.

40. Considero cedo para eu começar a pensar sobre como passar meu tempo na apo-

sentadoria.

을 42. Possuo um entendimento claro de como as pessoas aposentadas passam seu tem-

(1) po.

33. Sei que pessoas da minha faixa etária estão desenvolvendo novos modos de ocupar

seu tempo.

53. Há muitas coisas que eu poderia fazer com o meu tempo se eu fosse forçado a me aposentar hoje.

$\frac{\widetilde{\sigma}}{\alpha}$ 11. Considero importante desenvolver novas atividades para quando eu me aposentar.

21. Tenho metas específicas sobre como quero passar meu tempo na aposentadoria.

0,42

0,69

0,59

0,79

0,53

$3,04^{*}$

$3,19^{*}$

50. Possuo metas específicas sobre as atividades futuras que eu gostaria de realizar como um aposentado.

Nota. $N=982$, Principal Axis Fatorial, método direct oblimin. 1-Representações; 2-Decisões de tempo; 3-Decisões de pares; e 4-Preparação. *Itens da escala de objetivos, com média do item.

TABELA 5. Estrutura fatorial da EPPIA - Subescala planejamento psicossocial

\begin{tabular}{|c|c|c|c|c|c|c|}
\hline & Itens & $\alpha$ & 1 & 2 & 3 & 4 \\
\hline \multirow{12}{*}{ 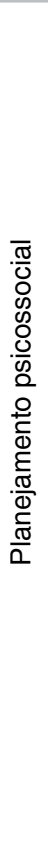 } & $\begin{array}{l}\text { 12. Frequentemente converso com minha família sobre as responsabilidades das pessoas } \\
\text { aposentadas. }\end{array}$ & & 0,95 & & & \\
\hline & 6. Falo frequentemente com pessoas aposentadas sobre como é estar aposentado. & & 0,81 & & & \\
\hline & $\begin{array}{l}\text { 44. Penso muito sobre minha responsabilidade como uma pessoa aposentada em minha } \\
\text { comunidade. }\end{array}$ & & 0,48 & & & \\
\hline & $\begin{array}{l}\text { 24. Frequentemente comparo minhas responsabilidades atuais com as que eu gostaria de } \\
\text { ter como uma pessoa aposentada. }\end{array}$ & & 0,41 & & & \\
\hline & $\begin{array}{l}\text { 31. Sei que pessoas da minha faixa etária estão se preparando para mudanças nas suas } \\
\text { responsabilidades quando se aposentarem. }\end{array}$ & 0,81 & 0,30 & & & \\
\hline & $\begin{array}{l}\text { 18. Considero importante me preparar para as mudanças em minhas responsabilidades } \\
\text { como uma pessoa aposentada. }\end{array}$ & & & 0,61 & & \\
\hline & $\begin{array}{l}\text { 30. Considero importante pensar sobre minhas responsabilidades futuras como aposenta- } \\
\text { do agora em vez de me preocupar no futuro. }\end{array}$ & & & 0,60 & & \\
\hline & $\begin{array}{l}\text { 49. Tenho um entendimento claro de como as responsabilidades das pessoas podem mu- } \\
\text { dar quando elas se aposentam. }\end{array}$ & & & 0,57 & & \\
\hline & 36. Tenho muitos interesses fora do trabalho a que eu gostaria de me dedicar. & 0,59 & & 0,40 & & \\
\hline & 20. Estou começando a me separar do trabalho. & & & & & 0,83 \\
\hline & 19. Estou reduzindo ou reduzirei em breve minhas horas de trabalho. & 0,75 & & & & 0,76 \\
\hline & 38. Possuo objetivos claros sobre os papéis futuros que pretendo ter como um aposentado. & & & $3,32^{*}$ & & \\
\hline
\end{tabular}

Nota. N=982, Principal Axis Fatorial, método direct oblimin. 1-Representações; 2-Decisões de tempo; 3-Decisões de pares; e 4-Preparação. *Itens da escala de objetivos, com média do item.

A validade discriminante visa verificar a capacidade da medida em discernir diferentes aspectos relacionados ao fenômeno observado. Para verificar esse indicador, foi realizada uma MANOVA com a variável demográfica "Empresa possui programa de orientação para a aposentadoria (OPA)?" e os escores gerais da EPPIA como variáveis dependentes. Procedeu-se primeiramente ao teste $M$ de Box para verificar a adequação dos dados à análise (Dancey \& Reidy, 2006). O teste foi significativo, $F(20,3018204)=43,091 ; p=0,002$, confirmando a violação na condição de homogeneidade. O teste de Levene, também utilizado para esse fim, apontou que todas as dimensões apresentaram quebra na homogeneidade: saúde $F(2,1283)=0,858$; $p=0,424$; finanças $F(2,1283)=1,429 ; p=0,240$; psicossocial $F(2,1283)=0,997 ; p=0,369$; e estilo de vida $F(2,1283)=0,158$; $p=0,854$. 
Para contornar as violações nos critérios de homogeneidade, realizou-se uma redução aleatória no banco para que as categorias da variável demográfica apresentassem número de participantes equivalentes. Com os grupos reduzidos, a configuração obtida foi: "sim", $N=351$ ( $71,1 \%$ homens [ $N=250]$ e idade $M=44,3$ anos [ $D P=9,8$ anos]); "não", $N=303$ (66,7\% homens [ $N=202]$ e idade $M=38,6$ [ $D P=10,1$ anos]); e "não sei", $N=328$ (57,6\% homens [ $N=189]$ e idade $M=36,9$ anos [ $D P=10,1$ anos]).

Os resultados da MANOVA mostram que ter serviço de OPA disponível na empresa influencia significativamente o planejamento nas quatro dimensões $(p<0,001)$. Médias e desvios-padrão são mostrados na Tabela 6. Participantes que relatam ter acesso ao programa de OPA apresentam maiores índices de planejamento em comparação aos que relataram não ter acesso ao programa ou não saber de sua existência. Na dimensão de planejamento psicossocial, participantes sem acesso a OPA possuem escores significativamente mais elevados que os que não sabem da existência do programa em suas organizações.

TABELA 6. Médias e desvios-padrão das MANOVAS da variável Empresa possui orientação para aposentadoria e as dimensões da EPPIA.

\begin{tabular}{lcccccccccc}
\hline & \multicolumn{3}{c}{ PS } & \multicolumn{2}{c}{ PF } & \multicolumn{3}{c}{ PE } & & PP \\
Sim & $M$ & $D P$ & $M$ & $D P$ & $M$ & $D P$ & $M$ & $D P$ \\
Não & $3,52_{\mathrm{a}}$ & 0,30 & $3,47_{\mathrm{a}}$ & 0,34 & $3,27_{\mathrm{a}}$ & 0,29 & $3,18_{\mathrm{a}}$ & 0,35 \\
Não Sei & $3,38_{\mathrm{b}}$ & 0,32 & $3,23_{\mathrm{b}}$ & 0,37 & $3,16_{\mathrm{ab}}$ & 0,31 & $2,85_{\mathrm{b}}$ & 0,37 & \\
\hline
\end{tabular}

Nota. Teste Post Hoc de Scheffe ( $p<0,001)$. Médias com mesmo subscrito não diferem entre si. PS= planejamento de saúde; PF= planejamento financeiro; $\mathrm{PE}=$ planejamento de estilo de vida; $\mathrm{PP}=$ planejamento psicossocial.

\section{DISCUSSÃO PARCIAL}

Os resultados indicam que o modelo neozelandês não se adequa plenamente à população brasileira, uma vez que houve divergência entre as subescalas do processo de planejamento, principalmente entre as etapas de representação e preparação. Isso indica dificuldade da amostra em definir um comportamento de planejamento da forma como previsto no modelo original, como um esforço para promover o bem-estar ao se aposentar (Muratore \& Earl, 2010).

A obtenção de piores índices de ajuste nos modelos novos, desenhados conforme orientações de Pasquali (1999) para a construção de escalas, é um indicativo da relevância da teoria de Friedman e Scholnick (1997) para a EPPIA. Considerando as indicações do Sistema de Avaliação dos Testes Psicológicos (Satepsi) (Resolução CFP 002/2003), os instrumentos traduzidos de outros idiomas devem ser adequados aos dados brasileiros considerando evidências de validade, precisão e os dados normativos para a população.

Ainda que o índice de $\chi^{2} / g l$ para os modelos de finanças, estilo de vida e psicossocial esteja acima do valor considerado ideal, esse é um parâmetro de análise de igual força em relação aos demais, sendo aceitável que se encontre acima do valor estimado ideal $(3,00)$ quando os demais indicadores estiverem ajustados (Byrne, 2010). Assim como no artigo original (Noone et al., 2010), algumas subescalas apresentaram alfas reduzidos, sendo um espaço para melhoria em pesquisas futuras. Próximos trabalhos utilizando a EPP1A podem elaborar novos itens para que as subescalas com apenas 2 itens sejam reforçadas e descrevam melhor o subfator em que estão alocadas (Pasquali, 2007, 2009).

Os resultados da MANOVA reforçam a importância dos programas de OPA, uma vez que a presença desse tipo de auxílio se mostrou discriminativa na realização de comportamentos de planejamento. Os programas são meios de transmitir informações sobre a aposentadoria, possibilitando a identificação de alternativas e a formação de planos, além de favorecer a troca de experiências e anseios (Costa \& Soares, 2009; Muniz, 1996). Quando não planejada, a aposentadoria pode impactar fortemente a vida dos indivíduos, possibilitando o surgimento e/ou agravamento de problemas psicológicos (Leandro-França et al., 2014).

A formatação do fenômeno da aposentadoria em termos de processo expõe a conexão entre a tomada de decisão e o planejamento. Para verificar essa configuração, decidiu-se realizar procedimentos estatísticos para verificar a validade preditiva das variáveis de estilos de tomada de decisão sobre o planejamento. 
Influência dos estilos de decisão no planejamento da aposentadoria - Indicadores de validade preditiva

Foram realizadas regressões hierárquicas múltiplas tendo como variáveis dependentes cada uma das escalas de planejamento. Em todas as análises, o bloco 1 foi composto pelas variáveis demográficas de controle: idade (em anos), sexo (1 masculino, 2 feminino), escolaridade (1 ensino superior, 2 sem ensino superior), estado civil ( 1 casado, 2 sem relacionamento) e interesse em voltar a trabalhar após se aposentar (bridge employment; 1 sim, 2 não). Foram incluídas todas as dimensões de estilo de decisão no bloco 2.

Todos os modelos foram significativos, demonstrando poder preditivo das variáveis demográficas e de estilo de decisão na explicação do planejamento da aposentadoria. Os valores de $\beta$ e $R^{2}$ estão disponíveis na Tabela 7 .

TABELA 7. Valores de beta das regressões de planejamento da aposentadoria

\begin{tabular}{|c|c|c|c|c|c|c|c|c|c|}
\hline & & \multicolumn{2}{|c|}{ Finanças } & \multicolumn{2}{|c|}{ Saúde } & \multicolumn{2}{|c|}{ Estilo de vida } & \multicolumn{2}{|c|}{ Psicossocial } \\
\hline & & $R^{2}$ & $\beta$ & $R^{2}$ & $\beta$ & $R^{2}$ & $\beta$ & $R^{2}$ & $\beta$ \\
\hline \multirow{5}{*}{ 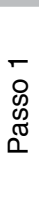 } & Idade & & $0,14^{\star *}$ & & $0,16^{\star \star}$ & & $0,21^{* *}$ & & $0,33^{* *}$ \\
\hline & Sexo & & $-0,01$ & & 0,04 & & $-0,01$ & & $-0,03$ \\
\hline & Escolaridade & 2,0 & $-0,07^{\star}$ & 3,0 & 0,01 & 4,0 & 0,01 & 10,4 & 0,01 \\
\hline & Estado civil & & 0,01 & & 0,04 & & $0,06^{*}$ & & 0,05 \\
\hline & Bridge emp. & & $-0,03$ & & $-0,06^{*}$ & & $-0,02$ & & $-0,03$ \\
\hline \multirow{11}{*}{ 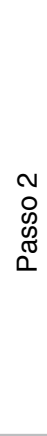 } & Constante & & $1,438^{\star *}$ & & $1,742^{\star \star}$ & & $0,927^{\star \star}$ & & $0,442^{* *}$ \\
\hline & Idade & & $0,13^{\star *}$ & & $0,14^{\star *}$ & & $0,18^{* *}$ & & $0,31^{\star *}$ \\
\hline & Sexo & & $-0,01$ & & 0,04 & & $-0,01$ & & $-0,03$ \\
\hline & Escolaridade & & $-0,07^{*}$ & & 0,02 & & 0,01 & & 0,01 \\
\hline & Estado civil & & 0,00 & & 0,04 & & 0,04 & & 0,03 \\
\hline & Bridge emp. & 13,7 & $-0,02$ & 18,1 & $-0,05^{\star}$ & 17,6 & $-0,01$ & 17,4 & $-0,02$ \\
\hline & E. Racional & & $0,29^{\star \star}$ & & $0,34^{\star *}$ & & $0,32^{\star \star}$ & & $0,20^{* *}$ \\
\hline & E. Intuitivo & & 0,05 & & 0,03 & & $0,06^{*}$ & & 0,03 \\
\hline & E. Dependente & & $0,07^{*}$ & & $0,07^{*}$ & & 0,04 & & 0,05 \\
\hline & E. Evitativo & & $-0,01$ & & $-0,07^{*}$ & & $0,06^{*}$ & & $0,09^{* *}$ \\
\hline & E. Espontâneo & & $0,06^{*}$ & & $-0,00$ & & $0,11^{*}$ & & $0,11^{* *}$ \\
\hline
\end{tabular}

Nota. Método Enter. ${ }^{* *}=p<0,001 ;{ }^{*}=p<0,05$; Brigde emp.= bridge employment, interesse em retornar ao trabalho após se aposentar.

No modelo de planejamento financeiro, os resultados indicam que, depois de controladas as variáveis do bloco 1, a idade, os estilos racional, dependente e espontâneo atuam como preditores significativamente positivos de maior planejamento, enquanto a escolaridade atua como preditor significativo e inverso. As variáveis explicam $13,7 \%$ da variância do modelo, $F(5,1272)=33,197 ; p<0,001$, indicando que ser mais velho, ter ensino superior completo e possuir estilo de tomada de decisão racional, dependente ou intuitivo contribuem positivamente para a economia e a readequação financeira.

Planejamento de saúde, após controle do bloco 1, obteve como preditores significativos e positivos a idade e os estilos de decisão racional e dependente, enquanto bridge employment e o estilo evitativo atuaram como preditores negativos. Com 18,1\% de variância explicada, o modelo foi significativo $(F[5,1272]=46,333 ; p<0,001)$ e expõe que ser mais velho e ter estilo de decisão racional ou dependente promovem melhor planejamento, enquanto ter interesse em retornar ao trabalho e ter estilo de tomada de decisão evitativo atuam negativamente para predizer maiores níveis de cuidados com a saúde física e mental.

Com relação ao planejamento de estilo de vida, as variáveis significativas explicam 17,6\% do modelo, $F(5,1272)=41,721 ; p<0,001$. Depois de controladas as variáveis demográficas, a idade e os estilos racional, intuitivo, evitativo e espontâneo contribuíram positivamente para predizer maior planejamento. Somente o estilo dependente não foi significativo, indicando que ser racional, agir de acordo com as próprias emoções, adiar a decisão e deixar que a vida se desenrole em seu próprio momento são opções mais benéficas que depender de terceiros para organizar a vida e a rotina na aposentadoria.

No modelo psicossocial, depois de controladas as variáveis demográficas, foi explicado 17,4\% da variância total, $F(5,1272)=23,558 ; p<0,001$. A idade e os estilos racional, evitativo e espontâneo foram significativos e positivos para prever maior planejamento psicossocial, indicando que ser mais velho, tomar decisões racionalmente, adiar as decisões ou decidir-se com o passar do tempo contribuem positivamente para o planejamento do desligamento do trabalho e a adequação aos novos papéis sociais da aposentadoria. 


\section{DISCUSSÃO GERAL}

O processo de adaptação de uma medida possui grande relevância científica, pois garante melhor adequação do instrumento às questões contextuais da amostra e culturais do país e da língua (Van Ommeren et al., 1999). Efeitos culturais devem ser minimizados ao máximo, mas levados em consideração principalmente no momento de apresentar a validade dos resultados (ITC, 2010). A EPP1A demonstrou, assim, atender aos requisitos para uso na população brasileira.

Conhecer os estilos de tomada de decisão mais associados ao melhor planejamento pode facilitar o desenvolvimento de ações para programas de OPA, estimulando diretamente a tomada de consciência e a definição de objetivos para o futuro (Leandro-França et al., 2014; Noone et al., 2010). Do ponto de vista teórico e empírico deste estudo, estilos de decisão mostraram ser preditores significativos do planejamento para aposentadoria. $\mathrm{O}$ estilo racional foi o único unânime nas quatro dimensões, reforçando que tendemos a traçar estratégias lógicas que influenciam nossas decisões (Gambetti, Fabbri, Bensi, \& Tonetti, 2008). Os resultados corroboram também o estudo clássico de Rubinton (1980), que pontua que indivíduos com estilo racional se desenvolvem melhor em situações que exigem elevada coerência e responsabilidade, como o planejamento financeiro (Lusardi \& Mitchell, 2007).

O estilo dependente apareceu como um bom preditor de planejamento, contrariando estudos de Rubinton (1980), Salo e Allwood (2011) e Allwood e Salo (2012). Os resultados demonstram a importância associada ao contato com o outro e com a troca de informações para o planejamento da aposentadoria. Ter maior pontuação no estilo dependente significa considerar as opiniões de outros e buscar auxílio para desenvolver novos comportamentos e estratégias de enfrentamento do desligamento do trabalho.

O estilo evitativo apareceu como preditor negativo para planejamento de saúde e finanças e positivo para estilo de vida e psicossocial, podendo contribuir ou dificultar os comportamentos de planejamento. Salo e Allwood (2011) observaram que o estilo evitativo se correlaciona com maior nível de "Autoestima baseada na performance", o que pode explicar a direção inversa do estilo entre os domínios de planejamento. Evitar decisões pode levar à não economia ou à não realização de exames preventivos, mas a natureza psicológica e social do estilo de vida e do planejamento psicossocial permite maior flexibilidade, uma vez que pode ser desgastante se focar excessivamente no futuro (Noone et al., 2009; Wang \& Shultz, 2010).

Como limitação tem-se a homogeneidade da amostra quanto à proveniência (um estado do Sudeste e empresas públicas). Futuros estudos devem obter dados de grupos mais diversos. Pode-se aprimorar a configuração da EPPlA quanto às subescalas que contam com apenas 2 itens e os alfas de Cronbach que possuem valores baixos. Finalmente, futuras pesquisas podem verificar as três etapas pré-transição para a aposentadoria, inserindo uma escala para avaliar percepções da aposentadoria e variáveis intervenientes.

Os dados brasileiros mostraram boa aderência à teoria do processo da aposentadoria, ligando de forma significativa a tomada de decisão ao planejamento. Ainda que a decisão de se aposentar, especificamente, seja influenciada por outros fatores que não somente os estilos de decisão (Appelt et al., 2011; Scott \& Bruce, 1995), os resultados deste trabalho servem como base para a realização de futuros estudos, que poderão explorar mais profundamente a relação entre decisão e planejamento na aposentadoria. O conhecimento do papel dos estilos de decisão no planejamento se mostra relevante, pois explicita a importância da porção individual de escolha. Futuros estudos podem buscar entender o papel desses estilos em programas de orientação para a aposentadoria, a fim de verificar se a obtenção de informações pode modificar o planejamento e também atuar de forma ativa para modificar os hábitos quanto ao assunto da aposentadoria.

\section{REFERÊNCIAS}

Adams, G. A., \& Rau, B. L. (2011). Putting off tomorrow to do what you want today: Planning for retirement. American Psychologist, 66(3), 180-192. doi:10.1037/a0022131

Allwood, C. M., \& Salo, I. (2012). Decision-making styles and stress. International Journal of Stress Management, 19(1), 34-47. doi:10.1037/a0027420

Antunes, M. H., Soares, D. H. P., \& Silva, N. (2013). Aposentadoria e contexto familiar: Um estudo sobre as orientações teóricas da produção científica. Perspectivas em Gestão \& Conhecimento, 3(número especial), 45-56.

Appelt, K. C., Milch, K. F., Handgraaf, M. J. J., \& Weber, E. U. (2011). The decision making individual differences inventory and guidelines for the study of individual differences in judgment and decision-making research. Judgment and Decision Making, 6(2), 252-262. doi:11/11218/jdm11218

Rev. Psicol., Organ. Trab., jan-mar 2016, vol. 16 num 1 
Babyak, M. A. (2004). What you see may not be what you get: A Brief, nontechnical introduction to overfitting in regression-Type models. Psychosomatic Medicine, 66, 411-421. doi: 0033-3174/04/6603-0411

Bonsang, E., \& Klein, T. J. (2012). Retirement and subjective well-being. Journal of Economic Behavior \& Organization, 83(3), 311-329. doi: 10.1016/j.jebo.2012.06.002

Byrne, B. (2010). Structural equation modeling with Amos: Basic concepts, applications and programming (2a ed.). New York: Routledge, Taylor \& Francis Group.

Costa, A. B., \& Soares, D. H. P. (2009). Orientação psicológica para a aposentadoria. Psicologia: Organizações e Trabalho, 9(2), 97-108.

Dancey, C. P., \& Reidy, J. (2006). Estatística sem matemática para psicologia (3a ed.). Porto Alegre: Artmed.

Feldman, D. C., \& Beehr, T. A. (2011). A three-phase model of retirement decision making. Am Psychol, 66(3), 193-203. doi: $10.1037 / \mathrm{a} 0022153$.

Friedman, S. L., \& Scholnick, E. K. (1997). The developmental psychology of planning: Why, how, and when do we plan? New York: L. Erlbaum Associates.

Gambetti, E., Fabbri, M., Bensi, L., \& Tonetti, L. (2008). A contribution to the Italian validation of the General Decision-making Style Inventory. Personality and Individual Differences, 44(4), 842-852. doi: 10.1016/j.paid.2007.10.017

Hambleton, R. K., \& Zenisky, A. L. (2011). Translating and adapting tests for cross-cultural assessments. In D. Matsumoto \& F. V. D. Vijver (Eds.), Cross-cultural research methods in psychology (pp. 46-74). New York: Cambridge University Press.

Hariri, H., Monypenny, R., \& Prideaux, M. (2013). Leadership styles and decision-making styles in an Indonesian school context. School Leadership \& Management, 34(3), 284-298.

Hox, J. J. (1994). Hierarchical regression models for interviewer and respondent effects. Sociological Methods \& Research, 22(2), 300-318.

International Test Commission (2010). International test commission guidelines for translating and adapting tests. Recuperado de http://www.intestcom.org.

Kim, J., Kwon, J., \& Anderson, E. (2005). Factors related to retirement confidence: Retirement preparation and workplace financial education. Financial Counseling and Planning, 12(2), 77-89.

Leandro-França, C., Murta, S. G., \& Villa, M. B. (2014). Efeitos de uma intervenção breve no planejamento para a aposentadoria. Revista Psicologia: Organizações e Trabalho, 14(3), 257-270.

Lei 8.842, de 4 de janeiro de 1994 (1994, 4 de janeiro). Dispõe sobre a política nacional do idoso, cria o Conselho Nacional do Idoso e dá outras providências. Recuperado de http://www.planalto.gov.br/ccivil_03/leis/L8842.htm

Lusardi, A., \& Mitchell, O. (2007). Financial literacy and retirement preparedness: Evidence and implications for financial education. Business Economics, 42(1), 35-44.

Marques, R. M., Batich, M., \& Mendes, A. (2003). Previdência social brasileira: Um balanço da reforma. São Paulo em Perspectiva, 17(1), 111-121. doi:10.1590/S0102-88392003000100011

Menezes, G. S., \& França, L. H. F. P. (2012). Preditores da decisão da aposentadoria por servidores públicos federais. Revista Psicologia: Organizações e Trabalho, 12(3), 315-328.

Moen, P. (1996). A life course perspective on retirement, gender, and well-being. Journal of Occupational Health Psychology, 1(2), 131-144.

Muniz, J. A. (1996). PPA: Programa de Preparação para o Amanhã. Estudos de Psicologia, 2(1), 198-204.

Muratore, A. M., \& Earl, J. K. (2010). Predicting retirement preparation through the design of a new measure. Australian Psychologist, 45, 98-111. doi: 10.1080/00050060903524471

Noone, J. H., Stephens, C., \& Alpass, F. M. (2009). Preretirement planning and well-being in later life: A prospective study. Research on Aging, 31(3), 295-317. doi:10.1177/0164027508330718

Noone, J. H., Stephens, C., \& Alpass, F. (2010). The process of retirement planning scale (PRePS): Development and validation. Psychological Assessment, 22(3), 520-531. doi:10.1037/a0019512

Pasquali, L. (1999). Instrumentos psicológicos: Manual prático de elaboração. Brasília: LabPAM \& IBAPP.

Pasquali, L. (2007). The validity of the psychological tests: Is it possible to find the way again? Psicologia: Teoria e Pesquisa, 23(special issue), 99-107.

Pasquali, L. (2009). Psicometria: Teoria dos testes na psicologia e na educação (3a ed.). Petrópolis: Vozes. 
Rafalski, J. C., \& De Andrade, A. L. (2014). Medidas psicológicas em contexto de aposentadoria (Relatório preliminar de pesquisa não publicado). Universidade Federal do Espírito Santo, Vitória.

Resolução CFP no002/2003, de 24 de março de 2003 (2003, 24 de março). Define e regulamenta o uso, a elaboração e a comercialização de testes psicológicos e revoga a Resolução CFP n 025/2001. Recuperado de https://www.policiamilitar. mg.gov.br/conteudoportal/uploadFCK/crs/File/documentos_normativos/resolucao_02_2003_cfp.pdf

Resolução nº466/2012, de 12 de dezembro de 2012 (2012, 12 de dezembro). Resolução do Conselho Nacional de Saúde. Recuperado de http://bvsms.saude.gov.br/bvs/saudelegis/cns/2013/res0466_12_12_2012.html

Rowlingson, K. (2002). Private pension planning: The rhetoric of responsibility, the reality of insecurity. Journal of Social Policy, 31(4), 623-642. doi: 10.1017/S0047279402006773

Rubinton, N. (1980). Instruction in career decision making and decision-making styles. Journal of Counseling Psychology, 27(6), 581-588.

Salo, I., \& Allwood, C. M. (2011). Decision-making styles, stress and gender among investigators. Policing: An International Journal of Police Strategies \& Management, 34(1), 97-119. doi:http://dx.doi.org/10.1108/13639511111106632

Scott, S. G., \& Bruce, R. A. (1995). Decision-making style: The development and assessment of a new measure. Educational and Psychological Measurement, 55(5), 818-831. doi:10.1177/0013164495055005017

Topa, G., Moriano, J. A., Depolo, M., Alcover, C.-M., \& Morales, J. F. (2009). Antecedents and consequences of retirement planning and decision-making: A meta-analysis and model. Journal of Vocational Behavior, 75(1), 38-55. doi:http://dx.doi. org/10.1016/j.jvb.2009.03.002

Van Ommeren, M., Sharma, B., Thapa, S., Makaju, R., Prasain, D., Bhattarai, R., \& de Jong, J. (1999). Preparing instruments for transcultural research: Use of the translation monitoring form with nepali-speaking bhutanese refugees. Transcultural Psychiatry, 36(3), 285-301.

Wang, M. (2012). Retirement: An introduction and overview of the handbook. In M. Wang (Ed.), The Oxford handbook of retirement (pp. 3-9). New York: Oxford University Press.

Wang, M., \& Shultz, K. S. (2010). Employee retirement: A review and recommendations for future investigation. Journal of Management, 36(1), 172-206. doi:10.1177/0149206309347957 